\title{
Comparison of the Luminal and Mucosa-Associated Microbiota in the Colon of Pigs with and without Swine Dysentery
}

\author{
Eric R. Burrough ${ }^{1 *}$, Bailey L. Arruda ${ }^{1}$ and Paul J. Plummer ${ }^{1,2}$ \\ 'Department of Veterinary Diagnostic and Production Animal Medicine, College of Veterinary Medicine, lowa State University, \\ Ames, IA, United States, ${ }^{2}$ Department of Veterinary Microbiology and Preventive Medicine, College of Veterinary Medicine, \\ lowa State University, Ames, IA, United States
}

\section{OPEN ACCESS}

Edited by:

Bradley L. Bearson, United States Department of

Agriculture, United States

Reviewed by: Ana M. Carvajal Urueña, Universidad de León, Spain

Tim Kåre Jensen,

Technical University of Denmark, Denmark

*Correspondence:

Eric R. Burrough

burrough@iastate.edu

Specialty section:

This article was submitted to Veterinary Infectious Diseases,

a section of the journal Frontiers in Veterinary Science

Received: 02 May 2017 Accepted: 09 August 2017 Published: 24 August 2017

Citation:

Burrough ER, Arruda BL and Plummer PJ (2017) Comparison of the Luminal and Mucosa-Associated Microbiota in the Colon of Pigs with and without Swine Dysentery.

Front. Vet. Sci. 4:139. doi: 10.3389/fvets.2017.00139
Colonic contents and mucosal scrapings from pigs inoculated with Brachyspira hyodysenteriae or Brachyspira hampsonii were collected at necropsy and classified as either positive $(n=29)$ or negative $(n=7)$ for swine dysentery (SD) based upon lesions and positive culture from the source pig. The microbiota in each sample was analyzed by bacterial census taking (16S rRNA gene sequencing). Procrustes analysis revealed similar clustering by disease classification with a relatively high M2 value $(0.44)$ suggesting differences in the microbiota between mucosal and luminal samples from the same pig. In both sample types, differences in richness and beta diversity were observed between disease statuses $(P \leq 0.014)$. The relative abundance of Brachyspirales, Campylobacterales, Desulfovibrionales, and Enterobacteriales was higher in pigs with dysentery for both mucosal scrapings and luminal samples while Clostridiales, Erysipelotrichales, and Fusobacteriales were significantly more abundant in the luminal contents only. For inoculated pigs that did not develop dysentery, Burkholderiales were more abundant in both sample types, Bacteroidales and Synergistales were more abundant in mucosal scrapings, and Lactobacillales and Bifidobacteriales were more abundant in luminal contents when compared with diseased pigs. Linear discriminant analysis of effect size revealed Brachyspira, Campylobacter, Mogibacterium, and multiple Desulfovibrio spp. as differential features in mucosal scrapings from pigs with dysentery while Lactobacillus and a Bifidobacterium spp. were differential in pigs without disease. These differential features were not observed in luminal samples. In summary, microbial profiles in both sample types differ significantly between disease states; however, evaluation of the mucosal microbiome specifically may be of higher value in elucidating bacterial mechanisms underlying development of SD.

Keywords: swine, microbial profiling, metagenomics, swine dysentery, Brachyspira hyodysenteriae, Brachyspira hampsonii

\section{INTRODUCTION}

Swine dysentery (SD) is characterized by severe mucohemorrhagic diarrhea and is associated with infection by strongly beta-hemolytic strains of Brachyspira hyodysenteriae, Brachyspira hampsonii, and Brachyspira suanatina (1). While these spirochetes are required for disease expression, SD only develops in pigs when one or more specific anaerobes are present in the microbiota $(2,3)$. 
Additionally, dietary modification can significantly alter the colonic microbiota of pigs and thereby increase or decrease expression of SD (4-6) suggesting that there may be specific microbial profiles that are permissive and resistant to $\mathrm{SD}$ expression.

With the advent of affordable next generation sequencing technology, research studies exploring microbial community profiles associated with heath and disease have flourished. For enteric disease states, many studies have focused on profiling the luminal contents or feces as these sample types are relatively easy to obtain; however, changes observed in these samples likely represent an indirect measure at best of what is happening at the mucosal surface where bacteria interact more intimately with the host and induce disease. In the case of SD, where there are profound changes in the colonic mucosa and where the etiologic agent can be readily visualized microscopically within the mucus layer, crypts, goblet cells, and epithelium (7), it seems logical to explore the microbiome directly associated with this biological niche in efforts to identify potential microbial biomarkers associated with disease susceptibility.

Accordingly, the microbial profiles of colonic contents and mucosal scrapings from pigs inoculated with $B$. hyodysenteriae or $B$. hampsonii were compared to determine differences between the microbiota of those pigs that developed SD following inoculation and those that did not. The a priori hypothesis of this study was that the microbial profiles in mucosal scrapings and luminal contents from the same pig differ significantly and that mucosal scraping profiles may reveal potential biomarkers of resistance and susceptibility to development of SD.

\section{MATERIALS AND METHODS}

\section{Colonic Samples}

Paired colonic content and mucosal scrapings from 36 commercial crossbred pigs receiving antibiotic-free rations and inoculated with either B. hyodysenteriae or B. hampsonii $\left(5.8 \times 10^{5}-1.2 \times 10^{6}\right.$ $\mathrm{CFU} / \mathrm{ml}$ once daily for 3 days) were collected at necropsy during a previous study (8). All animal procedures were approved by the Institutional Animal Care and Use Committee of Iowa State University (Log Number: 1-12-7283). Pigs were approximately 9 weeks old at the time of necropsy and were euthanized by barbiturate overdose within $72 \mathrm{~h}$ of SD development, which occurred between 6 and 14 DPI, or at the end of the study at 21 DPI. The spiral colon was exteriorized within approximately 5 min after death and luminal contents were collected from an incision at the apex of the spiral colon. A separate set of disinfected instruments was used for each pig and the luminal contents were collected into individual sterile $2.0 \mathrm{ml}$ cryogenic vials (Corning Inc., Corning, NY, USA) and were snap frozen in liquid nitrogen. The apex of the spiral colon was then opened to reveal the mucosa, residual contents were gently removed from the surface, and a mucosal scraping was obtained using the blade of a post mortem knife. Mucosal scrapings were then transferred into individual sterile polystyrene snap-cap tubes (Corning Inc., Corning, NY, USA), refrigerated for $2-4 \mathrm{~h}$, and then frozen and retained at $-80^{\circ} \mathrm{C}$ until further processing for use in this study. The paired samples were classified as either positive $(n=29)$ or negative $(n=7)$ for SD based on the presence of mucohemorrhagic diarrhea, appropriate microscopic lesions in fixed colonic tissues (neutrophilic infiltration of the lamina propria and increased mucosal thickness), and recovery of a strongly hemolytic Brachyspira by selective anaerobic culture from the source pig. Brachyspira cultivation was performed anaerobically using selective agar containing spiramycin, rifampin, vancomycin, colistin, and spectinomycin with incubation for at least 6 days.

\section{DNA Purification}

Colonic contents and mucosal scrapings were processed for DNA extraction using the Qiagen DNA Stool MiniKit following the manufacturer's recommendations. Mucosal scrapings were then processed through the Qiagen PCR Purification Kit (Qiagen part 28106) following the manufacture's recommendations to remove some residual extraction buffer salts that remained after the original purification. Following DNA purification, samples were screened for DNA concentration and purity using a Nanodrop DNA Flouremeter and the Qubit fluorometer (Life Technologies, Grand Island, NY, USA) and DNA was stored at $-80^{\circ} \mathrm{C}$ prior to downstream processing.

\section{$16 S$ Sequencing}

DNA from the extracted colonic content samples and paired mucosal scrapings were submitted to Argonne National Laboratory-Institute for Genomics and Systems Biology Next Generation Sequencing Core (http://ngs.igsb.anl.gov/) for metagenomic analysis using the $\mathrm{V} 4$ region of the bacterial $16 \mathrm{~S}$ rRNA gene. All samples were processed by the routine methodology of the core laboratory. Briefly, amplicons were synthesized using a universal $16 \mathrm{~S}$ forward primer $(515 \mathrm{~F})$ and individual unique Golay barcoded reverse primers (806R) as described (9). Appropriate positive and negative controls were included by the sequencing facility. Sample library DNA concentrations were quantified and samples were pooled with equal amounts of DNA. The pooled libraries were cleaned up with the MO-BIO UltraClean PCR Clean-Up Kit and the concentration was then diluted to $2 \mathrm{nM}$. For each sample type, a single flow cell lane containing 100 samples (the 36 samples of this report and 64 additional samples) of 300-bp paired end sequences was run on the Illumina MiSeq.

\section{Metagenomic Data Analysis}

Forward and reverse reads from the paired end sequencing were first merged using the fastq.join script. Qiime 1.8 was then used for additional data analysis. De-multiplexing and quality filtering were then performed using the split_libraries_fastq.py script. The pick_reference_otus_through_otu_table.py script was used for operational taxonomic unit (OTU) calling and taxonomic assignment was performed based on the greengenes database (10). All libraries were adjusted to 47,000 reads for luminal contents and 56,000 reads for mucosal scrapings to avoid potential interpretation errors due to variable sampling depth. Comparisons of specific OTUs within groups were made at the phylum, order, and genus level and only those OTUs detected in at least 25\% of samples were included in the analysis. Biological effect sizes were estimated using the linear discriminant analysis effect size 
(LEfSe) method (11) and a Procrustes analysis was performed to compare profiles generated from paired contents and scrapings in individual pigs (10). For colonic mucosal scraping samples, a CoVennTree (Comparative weighted Venn Tree) analysis (12) was performed to assess differences in the microbial population structure between pigs with SD and those inoculated pigs that did not develop SD.

\section{Statistical Analyses}

Statistical output was generated by Qiime 1.8. Alpha diversity (chao1) was compared using a non-parametric two sample t-test with 999 Monte Carlo permutations. Beta diversity (BrayCurtis dissimilarity) was compared using a two-sided student's two-sample $t$-test with Bonferroni correction. The frequency of detection (group significance) of specific OTU calls within groups was compared using a Kruskal-Wallis non-parametric analysis of variance followed by correction for multiple comparison using the Benjamini and Hochberg False Discovery Rate (FDR) method (13). A FDR of 5\% was utilized to determine significance. Firmicutes:Bacteroidetes ratios were calculated based upon the relative abundance percentages reported in Qiime and were compared using a two-sided student's two-sample $t$-test. For outputs where $P$-values were derived, statistical significance was defined as $P<0.05$.

\section{RESULTS}

In both luminal content samples and mucosal scrapings, there were significant differences in richness (chao1; $P=0.014$ and $P=0.001$, respectively; Figure 1$)$ and beta diversity $(P<0.001$, both sample types) between samples from pigs with and without SD. The Procrustes analysis revealed generally similar spatial clustering by disease classification yet with a relatively high $M^{2}$ value (0.44).
At the phylum level, the relative abundance of Firmicutes was greater in the luminal samples of pigs with SD whereas Proteobacteria and Fusobacteria were more abundant in the mucosal scrapings of diseased pigs relative to those not developing disease (Figure 2). Bacteroidetes and Synergistetes were more abundant in scrapings from pigs that did not develop SD. The Firmicutes:Bacteroidetes ratios in luminal content samples were significantly higher in pigs with SD (mean $0.508 \pm 0.226$ ) relative to inoculated pigs that did not develop disease (mean $0.250 \pm 0.134)(P=0.001)$.

At the order level, the relative abundance of Brachyspirales, Campylobacterales, Desulfovibrionales, and Enterobacteriales was higher in pigs with SD for both mucosal scrapings (Figure 3) and luminal samples while Clostridiales, Erysipelotrichales, and Fusobacteriales were more abundant in the luminal contents only. For inoculated pigs that did not develop SD, Burkholderiales were more abundant in both sample types, Bacteroidales and Synergistales were more abundant in scrapings, and Lactobacillales and Bifidobacteriales were more abundant in luminal contents only.

At the genus level, the relative abundance of Brachyspira, Campylobacter, Fusobacterium, and Mogibacterium was higher in pigs with SD for both mucosal scrapings and luminal samples while Desulfovibrio were more abundant in scrapings only and Flexispira were more abundant in the luminal contents only. For inoculated pigs that did not develop SD, Streptococcus and Ruminococcus were more abundant in both sample types while Prevotella and Roseburia were more abundant in scrapings only. Brachyspira OTUs were detected in 22 of 29 luminal content samples and 29 of 29 mucosal scrapings from pigs with $\mathrm{SD}$ versus none (0 of 7 ) of the luminal content samples and only one mucosal scraping sample from inoculated pigs that did not develop SD. When detected, the relative abundance of Brachyspira was low averaging $0.02 \%$ in mucosal scrapings
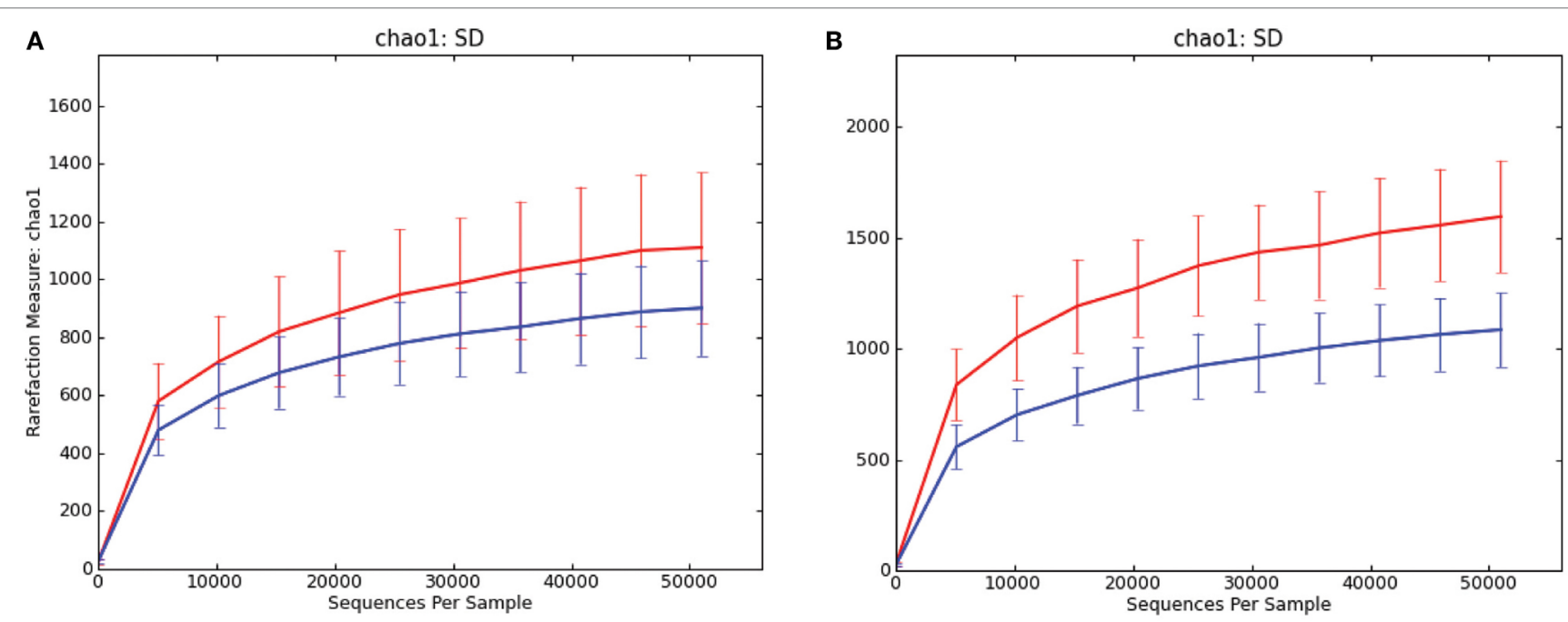

FIGURE 1 | Rarefaction curves comparing alpha diversity (chao1) of microbiota samples from pigs with swine dysentery (blue lines) and without dysentery (red lines) after experimental inoculation. Samples of both colonic luminal contents $\mathbf{( A )}$ and colonic mucosal scrapings (B) reveal significant differences in richness between disease states $(P=0.014$ and $P=0.001$, respectively). 


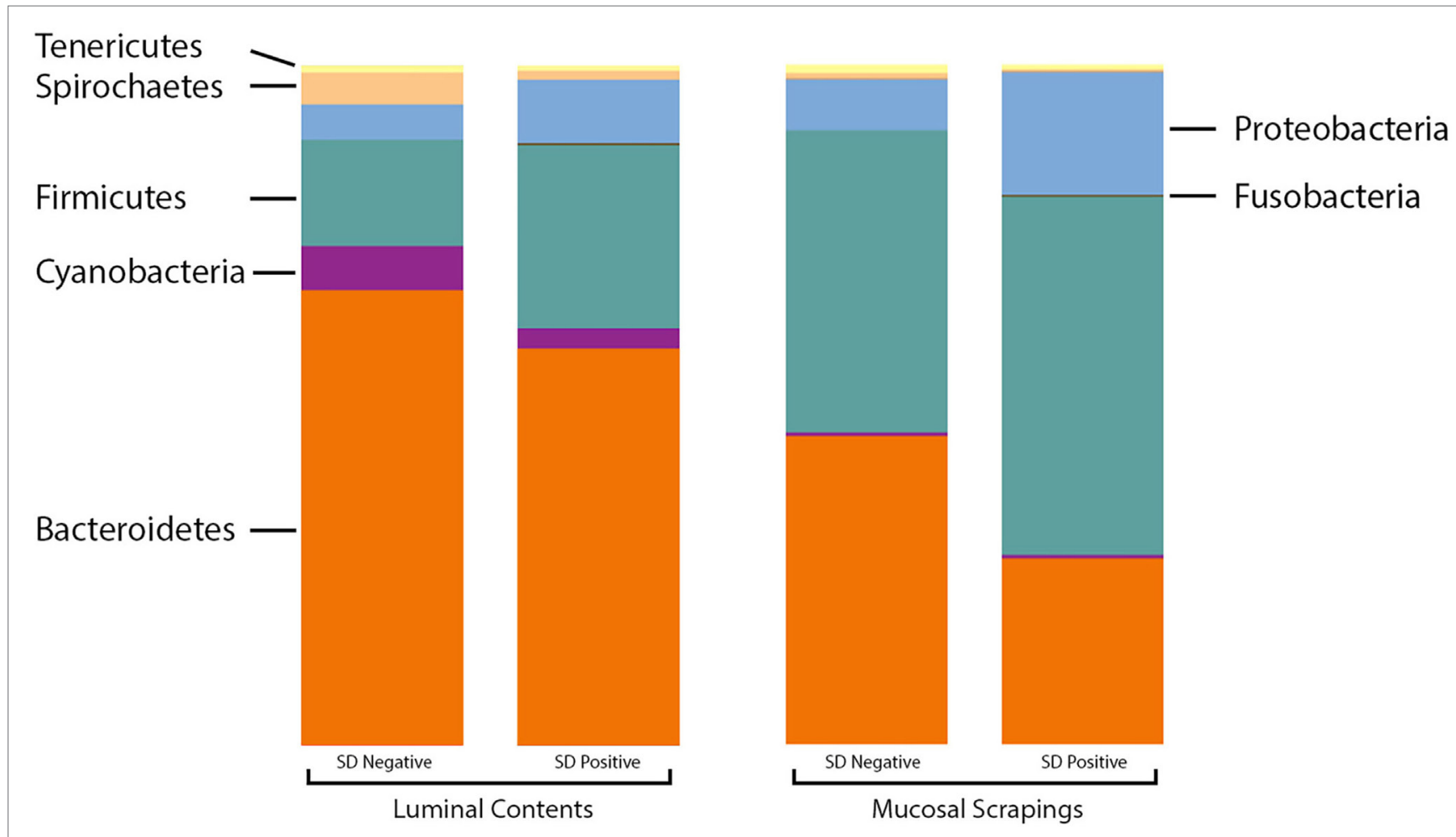

FIGURE 2 | Stacked bar charts representing proportional abundance of major phyla in microbiota from 9-week-old pigs with and without swine dysentery (SD). The bars on the left reflect comparison of colonic luminal content samples and bars at the right refelct comparison of colonic mucosal scrapings. The

Firmicutes:Bacteroidetes ratios in luminal content samples were significantly higher in pigs with SD relative to inoculated pigs that did not develop disease $(P=0.001)$

and $0.006 \%$ in luminal content samples. A CoVennTree analysis of the microbiota in mucosal scraping samples from pigs with and without SD reveals the relative size (number of reads) and similarity (degree of overlap) of detected taxa (Figure 4).

LEfSe revealed Brachyspira, Campylobacter, Mogibacterium, Oscillospira, Anaerotruncus, and multiple Desulfovibrio spp. as differential features in mucosal scrapings from pigs with SD while Lactobacillus, Roseburia, Synergistales, a Bifidobacterium spp., and a specific Desulfovibrio spp. were differential in pigs without disease (Figure 5). The Plot One feature of LEfSe provides a more detailed comparison between samples (Figure 6).

The Brachyspira OTUs detected were a $100 \%$ match over the region compared to $B$. hyodysenteriae and $B$. hampsonii. The predominant Campylobacter OTU detected had high nucleotide sequence homology (100\%) based on a blast search to Campylobacter hyointestinalis and the predominant Desulfovibrio OTUs detected in SD pigs had high nucleotide sequence homology (95\%) based on a blast search to Desulfovibrio desulfuricans. One specific Desulfovibrio OTU was a differential feature in LEfSe for pigs without $\mathrm{SD}$, and this OTU had high nucleotide sequence homology (99\%) based on a blast search to Desulfovibrio spp. Marseille-P2429.

\section{DISCUSSION}

While Procrustes analysis of the microbiota revealed similar clustering of samples by disease status, the $M^{2}$ value suggests the data sets are not a particularly good fit and that differences exist between the microbiota of the colonic contents and scrapings from the same pig. This is consistent with a recent report where microbial profiles from the cecal mucosa and cecal content of pigs differed significantly with luminal content samples being more diverse (14). While not unexpected, this paired analysis emphasizes that exploration of both sample types may be warranted when studying specific intestinal diseases and exploration of the mucosal microbiome specifically may yield more specific information regarding those species in more intimate contact with the host.

Significantly higher Firmicutes:Bacteroidetes ratios were observed in the luminal contents of pigs with SD relative to those without disease, which is consistent with previous studies where higher ratios have been reported in both dogs and humans with diarrhea regardless of cause $(15,16)$. Altered ratios were also reported in the fecal microbiota from pigs with SD after inoculation with $B$. hampsonii (17); however, in this previous investigation, there were no statistically significant differences detected in the microbiota of feces from pigs that did and did not develop mucohemorrhagic diarrhea. Possible explanations for the discord between this previous investigation and the current study include sequencing depth (samples were normalized to 1,000 reads in the previous investigation versus 47,000 and 56,000 in the current study), examination of feces versus luminal contents and mucosal scrapings, limitation to phylum level analysis in the previous report versus order and genus level herein, and the inclusion 


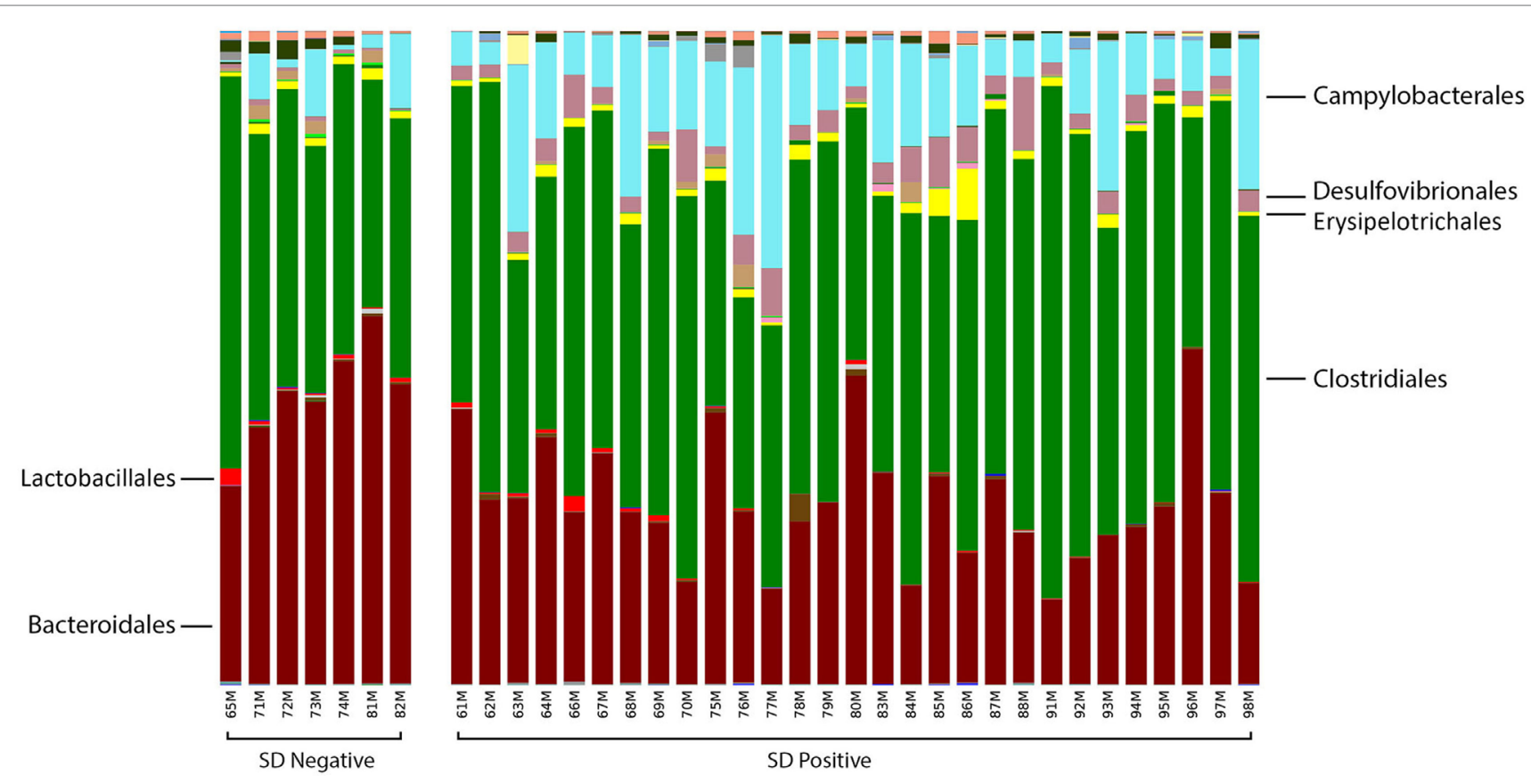

FIGURE 3 | Stacked bar charts representing proportional abundance of major bacterial orders in the microbiota of colonic mucosal scrapings from 9-week-old pigs with or without swine dysentery following experiemntal inoculation with Brachyspira hyodysenteriae or Brachyspira hampsonii. Bars represent results of $16 \mathrm{~S}$ rRNA gene sequencing followed by assignment of operational taxonomic units. Pigs that developed dysentery had an increased relative abundance of Brachyspirales (total percentage too small to be visible in this graph), Campylobacterales, and Desulfovibrionales, whereas those pigs that did not develop disease had increases in abundance of Bacteroidales and Synergistales (not visible in this graph). A false discovery rate of $5 \%$ was used to determine significance.

of sham-inoculated controls in the previous investigation as it is unknown if the profiles from those pigs would have been susceptible or resistant to disease expression.

In the study of this report, both luminal contents and mucosal scrapings from inoculated pigs with SD had significant differences in the relative abundance of multiple bacteria at the order level when compared with samples from inoculated pigs that did not develop disease. Previous investigations have revealed that when specific anaerobes, such Fusobacterium necrophorum, are present in the microbiota, pigs are rendered susceptible to SD development following inoculation with B. hyodysenteriae (3). Consistent with this finding, Fusobacteria were more abundant in luminal contents of pigs that developed SD after inoculation. A significant increase in Fusobacterium has also been reported in fecal samples from weaned pigs with diarrhea associated with porcine epidemic diarrhea virus infection (18) as well as in nursing pigs with non-specific diarrhea (19) suggesting that this finding may also be reflective of dysbiosis associated with certain types of diarrhea.

Campylobacter (Vibrio) coli has been historically associated with pigs with SD and at one point was considered a potential etiologic agent prior to the identification of Treponema hyodysenteriae (now B. hyodysenteriae) (20). In an investigation of fecal samples from pigs with diarrhea of various causes, a positive Campylobacter culture was obtained from all pigs with a laboratory diagnosis of Brachyspira-associated disease suggesting a potential interrelationship between these bacteria in the large intestine of pigs (21). Not surprisingly, this association is clearly supported by the data of the current investigation where Campylobacterales were more abundant in all samples types from pigs with SD. Campylobacter spp. are commonly present in the mucosa-associated microbiota of the porcine large intestine (14), and their enrichment in SD may also reflect perturbation of the normal mucosal community after colonization of this niche by Brachyspira.

LEfSe further revealed that both Brachyspira and Desulfovibrio spp. were differential features at the mucosal surface of pigs with $\mathrm{SD}$, which is of note as Desulfovibrio spp. are sulfate reducing bacteria with the potential to degrade the sulfated mucins that comprise part of the mucus barrier and utilize mucin as a substrate (22). Indeed, a reduction in sulfated mucins has been previously reported in pigs with acute SD (23) and this breakdown in the organization of colonic mucus has been shown to provide more mucin-binding sites for B. hyodysenteriae (24). Desulfovibrio spp. are also consistently increased in colonic biopsies from people with ulcerative colitis (25) and it is proposed that these bacteria may contribute to colitis through the production of toxic hydrogen sulfide, a byproduct of metabolism of sulfated mucins (26). $D$. desulfuricans has increased affinity to mucins from patients with ulcerative colitis; however, strain-specific differences in mucin binding capacity have been observed with some strains having reduced affinity relative to the type strain (22). This is consistent with the findings of the present report where a majority of detected Desulfovibrio OTUs were differential for scrapings from pigs with SD while a single OTU was differential for pigs that did not develop SD. Brachyspira accounted for an extremely small 


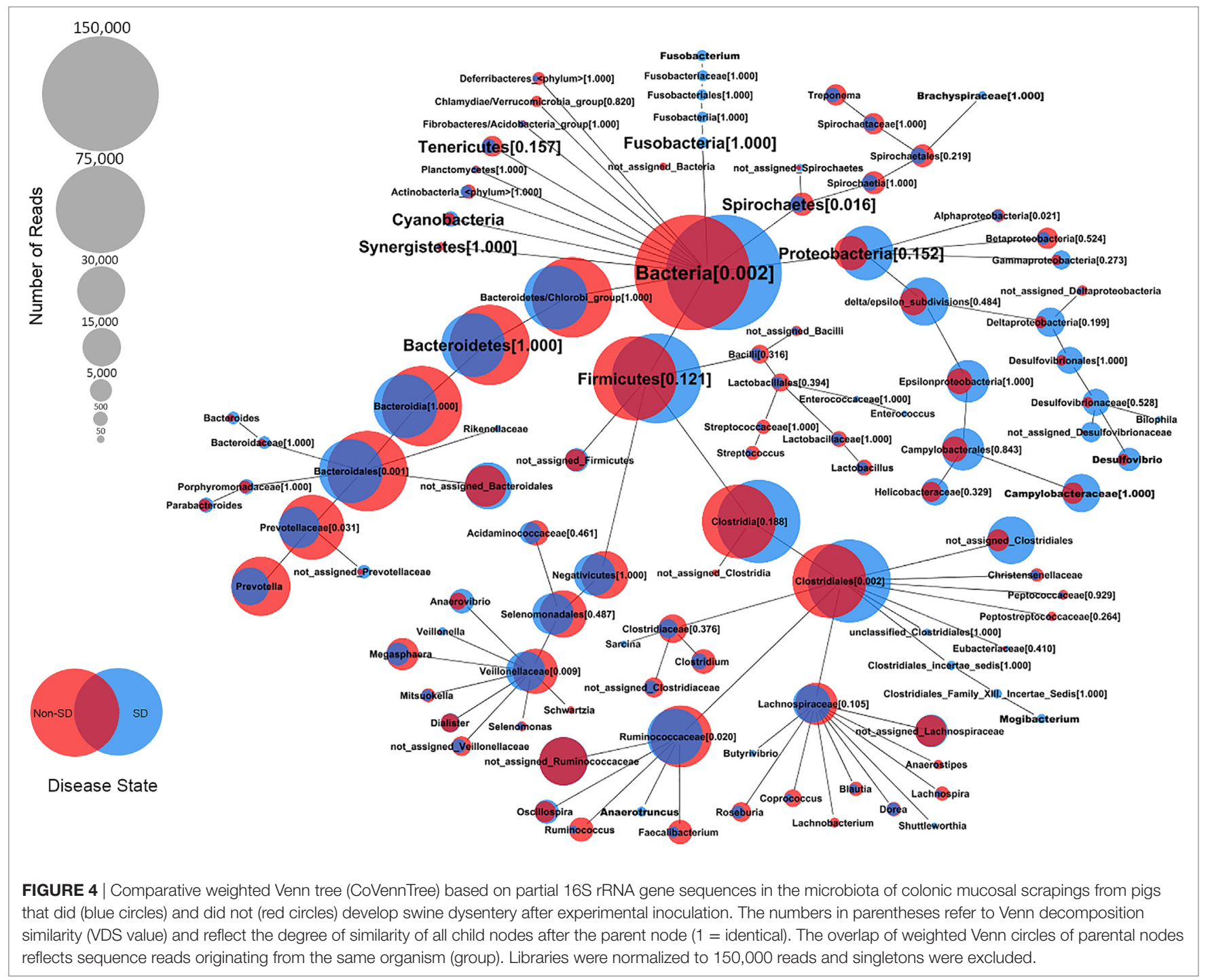

proportion of the total microbiota $(\leq 0.02 \%)$, even in mucosal scrapings from pigs with acute SD.

Mogibacterium, Anaerotruncus, and Oscillospira were also differential features of the microbiota from pigs with SD; however, little has been reported regarding the role of these bacteria in animals. Mogibacterium is an oral bacterium commonly associated with human periodontal disease and has been shown to have greater relative abundance in the fecal microbiota of people with adenomatous polyps versus those without (27). Anaerotruncus is a recently described organism that has been detected in human feces and a single case of human bacteremia (28). Oscillospira, on the other hand, is an anaerobic bacterium that is reduced in several human inflammatory conditions (29) and thus its association with SD seems contradictory. Further investigation into the role of these bacteria in health and disease in livestock species is needed.

Lactobacillus and a Bifidobacterium spp., two genera commonly investigated as probiotic agents, were differential features in the mucosa of inoculated pigs that did not develop SD. These and other lactic acid-producing bacteria can reduce luminal $\mathrm{pH}$, produce bacteriocins, and protect biological niches through competitive exclusion. Lactobacillus spp. exhibit significant differences in their ability to bind mucin and to exclude Salmonella Typhimurium and Escherichia coli in vitro, suggesting certain species may be more beneficial in reducing pathogen binding in vivo (30). A significant reduction in Lactobacillus spp. was observed in pigs consuming $30 \%$ DDGS relative to pigs fed a standard corn-soy diet (4), and pigs consuming this same diet (30\% DDGS) had a shorter time to onset of SD following $B$. hyodysenteriae infection relative to controls (8) further suggesting decreased Lactobacillus may be one potential biomarker of susceptibility to SD development. Reductions in Lactobacillus have also been reported in nursing pigs with diarrhea of unspecified cause (19). Lactobacilli and bifidobacteria produce lactate, which in turn can be used by certain butyrate-producing bacteria, such as Megasphaera (6), and increased levels of this short chain fatty acid may improve colonic health. 


\section{SD $\quad S D$ \\ $\square \mathrm{Neg} \square$ Pos}

$\begin{array}{lllll}1 & 1 & 1 & 1 & 1\end{array}$ Bacteria.Firmicutes.Clostridia.clostridialeș. Ruminocóccaceae Bacteria.Proteobacteria.Delt [..jbrionaceaje.Desulfovibrio.s Bacteria.Firmicutes.Clostrid [..]Rُuminococicaceae.Ościllospira Bacteria.Firmicutes.çlostrid [..]nococcacẹae.Oscillọspira.s Bacteria.Proteobacteria:Epsi [.. ]ylobacteracéae.Campỵlobacter Bacteria.Protéobacteriạ..Epsi [.. ]açterales.Cạmpylobaçteraceae Bacteria.Firmicutes.Clośtrid [..]uminococcacieae.Anaeŕotruncus Bacteria.Firmicutes.Clọstrid [.. ]oçoccaceae.Anaerotrüncus.s Bacteria.Firmicutes.Clostrid [..]ibacteriacéae_.Mogibacterium Bacteria.Firmicutes.Ciostrid [..]țeriaceae_;Mogibactẹrium.s

Bacteria.Firmicutes.Clostrid [...]tridiales. Mogibactèriaceae Bacteria.Spirochaetes._Brach [..]chyspirales_.Brachyspiraceae Bacteria.Spirochaetes.'Brach [..]'Brachyspiraceae.Brachyspira Bacteria.Spirochaetes.;Brach [..]chyspiraceeae.Brachyspira.s Bacteria.Spirochiaetes._Brachyspiraè_-_Brachyispirales
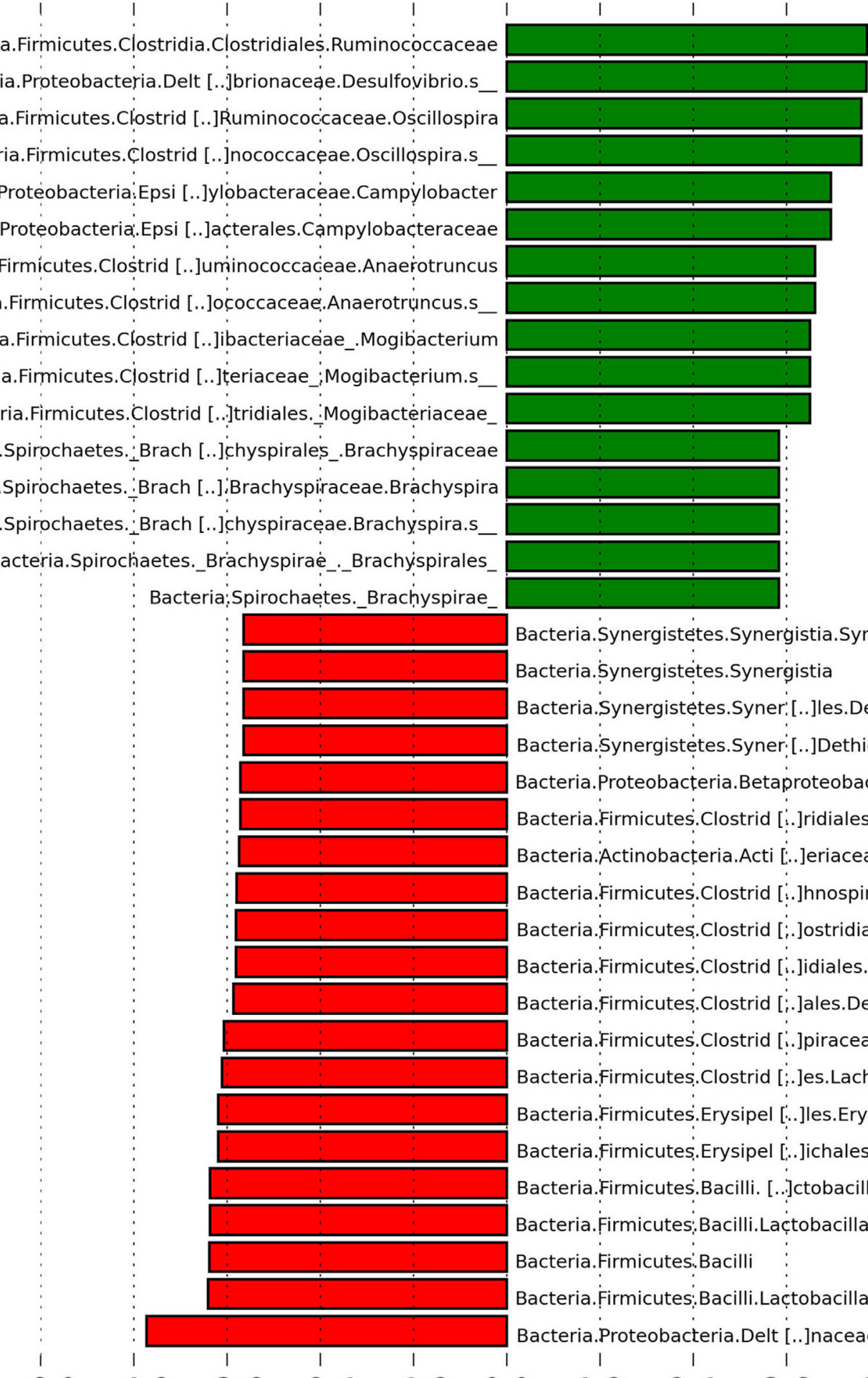

Bacteria.Synergistetes.Synergistia.Synergistales Bacteria.Śynergistètes.Synergistia Bacteria.Synergistẹtes.Syner', [..]les.Dethiosulfovibrionaceae. Bacteria.Synergistètes.Syner:[..]Dethiosulfovibrionaceae..s Bacteria.Proteobacțeria.Betaproteobacțeria.Burkholderiales Bacteria.Firmicutes.Clostrid ['..]ridiales.Dehalobacteriaceae. Bacteria.Actinobacteria.Acti [:..] ]eriaceaè.Bifidobacterium.s_ Bacteria.Firmicutes.Clostrid ['.].]hnospiráceae.Coprococcus.s Bacteria.Firmicutes.'Clostrid [';. ]ostridiales. Ruminococcaceae. Bacteria.Firmicutes. Clostrid ['.] ]idiales. Ruminococcaceae..s_ Bacteria.Firmicutes. Clostrid [i. ]ales.Dehalobacteriaceae..s_ Bacteria.Firmicutes.Clostrid [i..]piraceaé.Roseburia.s_faecis Bacteria.Firmicutes..Clostrid [:.].]es.Lachṇospiraceae. Roseburia Bacteria.Firmicutes. Erysipel [’..]les.Erysipelotrichaceae..s_ Bacteria.Firmicutes.. Erysipel ['.. . ]ichales. Érysipelotrichaceae. Bacteria.Firmicutes.Bacilli. [...]ctobacillaceae.Lactobacillus Bacteria.Firmicutes:Bacilli.Laçtobacillalẹs.Lactobacillaceae Bacteria.Firmicutes'Bacilli Bacteria.Firmicutes:Bacilli.Laçtobacillalès Bacteria.Proteobacteria.Delt [..]naceae.'Desulfovibrio.s_D168 
FIGURE 5 | Continued

Histogram of linear discriminant analysis (LDA) scores computed by LEfSe revealing differentially abundant taxa in the microbiota of mucosal scrapings from pigs with or without swine dysentery (SD) following experimental inoculation with Brachyspira hyodysenteriae or Brachyspira hampsonii. Brachyspira, Campylobacter, Mogibacterium, Anaerotruncus, Oscillospira, and multiple Desulfovibrio spp. were differential features in mucosal scrapings from pigs with SD while Lactobacillus, Roseburia, Synergistales, a Bifidobacterium spp., and a specific Desulfovibrio spp. were characteristic of samples from those pigs that were resistant to infection and disease development.

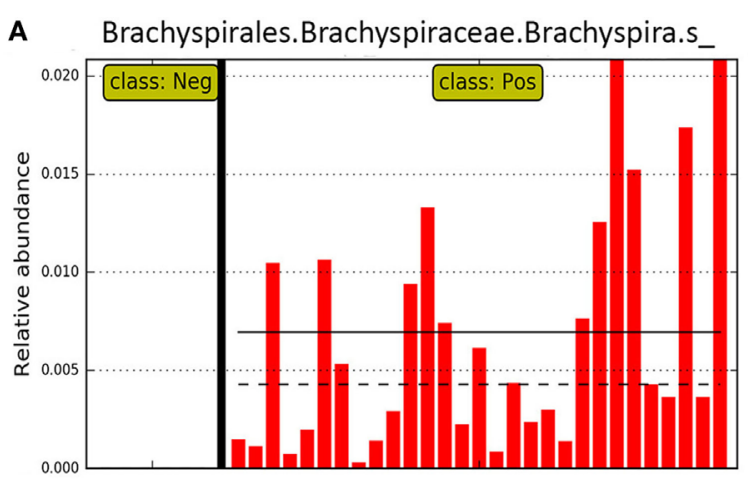

\section{C}

Campylobacterales.Campylobacteraceae.Campylobacter

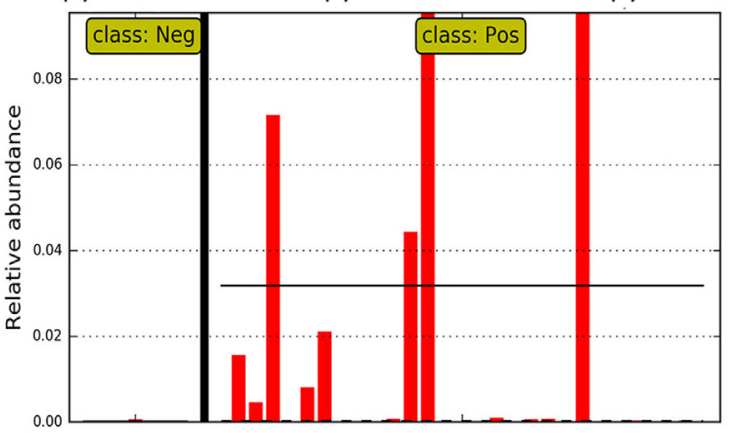

$\mathbf{E}$

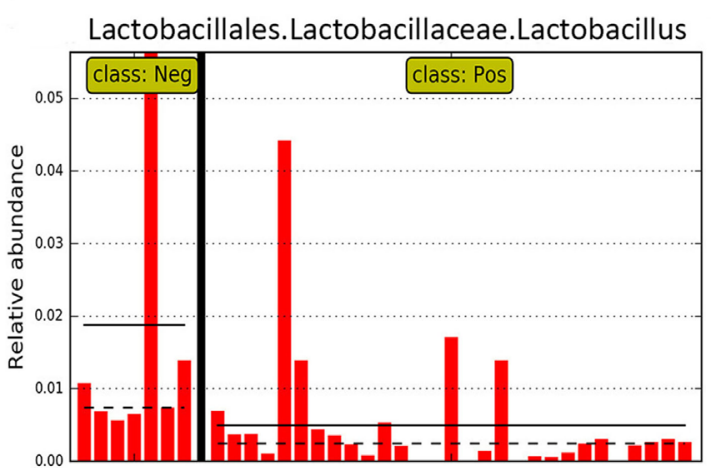

\section{B Desulfovibrinonales.Desulfovibrionaceae.Desulfovibrio.s_}

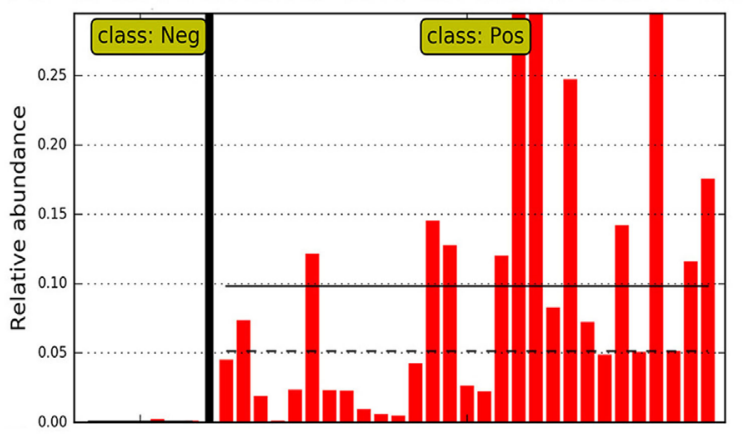

D

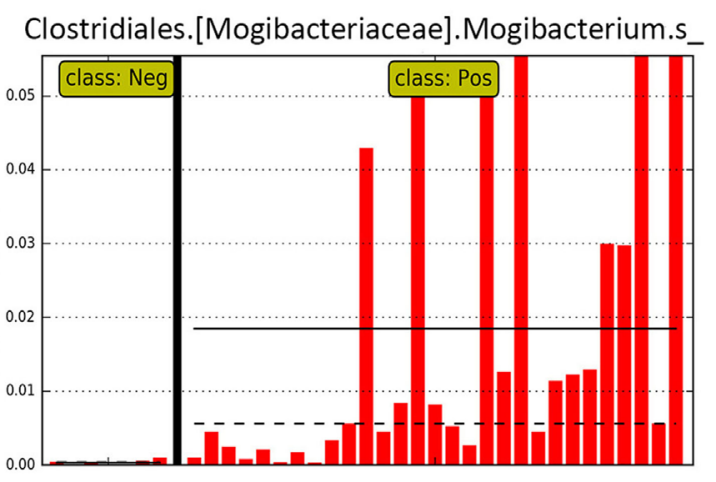

$\mathbf{F}$

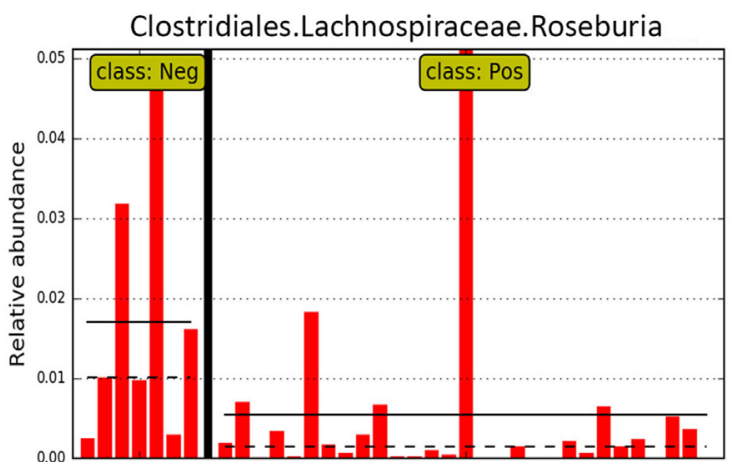

FIGURE 6 | Bar graphs representing relative abundance in individual pig mucosal scraping samples of specific genera identified as differential features in LEfSE for pigs with (class: Pos) and without (class: Neg) swine dysentery (SD) after experimental inoculation. Solid black horizontal lines represent the mean abundance within each class. Panels (A-D) represent genera that were differential features of the microbiota from pigs with SD, whereas panels (E,F) represent two genera that were differential for the mucosal microbiota from pigs without SD.

Butyrate is the primary energy source for colonocytes and has also been shown to modulate the immune system, suppress cancer, reduce oxidative stress, and modulate intestinal motility modulation (31). In the current study, Roseburia, a butyrateproducing anaerobe commonly detected in the pig colon (14), was enriched in mucosal scrapings of inoculated pigs that did 
not develop SD, and Roseburia faecis specifically was a differential feature of the microbiota from pigs without disease. This is consistent with previous reports suggesting Roseburia as a biomarker of a healthy gut (32).

In summary, the microbial profiles of mucosal scrapings were different than those in adjacent luminal contents but reveal similar clustering by disease phenotype. Mucosal scraping samples offered better detection of agents of SD and are likely a more direct assessment of the ecological niche of SD. Evaluation of the microbiota in mucosal scrapings revealed numerous potential biomarkers of disease (Desulfovibrio, Campylobacter, Mogibacterium, and Fusobacterium) and absence of disease development (Lactobacillus, Bifidobacterium, and Roseburia) following inoculation with agents of SD; however, it remains to be determined if these differences are a consequence of SD or related to cause. Investigation into the potential role of these bacteria in the pathogenesis of SD or in resistance to disease expression and thereby the utility of their detection in the microbiota in evaluating potential prebiotic approaches to SD control is warranted.

\section{REFERENCES}

1. Burrough ER. Swine dysentery: etiopathogenesis and diagnosis of a reemerging disease. Vet Pathol (2017) 54:22-31. doi:10.1177/0300985816653795

2. Meyer RC, Simon J, Byerly CS. The etiology of swine dysentery. III. The role of selected gram-negative obligate anaerobes. Vet Pathol (1975) 12:46-54. doi:10.1177/030098587501200107

3. Whipp SC, Robinson IM, Harris DL, Glock RD, Matthews PJ, Alexander TJ. Pathogenic synergism between Treponema hyodysenteriae and other selected anaerobes in gnotobiotic pigs. Infect Immun (1979) 26:1042-7.

4. Burrough ER, Arruda BL, Patience JF, Plummer PJ. Alterations in the colonic microbiota of pigs associated with feeding distillers dried grains with solubles. PLoS One (2015) 10:e0141337. doi:10.1371/journal.pone. 0141337

5. Hansen CF, Hernández A, Mansfield J, Hidalgo Á, La T, Phillips ND, et al. A high dietary concentration of inulin is necessary to reduce the incidence of swine dysentery in pigs experimentally challenged with Brachyspira hyodysenteriae. Br J Nutr (2011) 106:1506-13. doi:10.1017/S000711451100208X

6. Mølbak L, Thomsen LE, Jensen TK, Bach Knudsen KE, Boye M. Increased amount of Bifidobacterium thermacidophilum and Megasphaera elsdenii in the colonic microbiota of pigs fed a swine dysentery preventive diet containing chicory roots and sweet lupine. J Appl Microbiol (2007) 103:1853-67. doi:10.1111/j.1365-2672.2007.03430.x

7. Burrough ER, Wilberts BL, Bower LP, Jergens AE, Schwartz KJ. Fluorescent in situ hybridization for detection of "Brachyspira hampsonii" in porcine colonic tissues. J Vet Diagn Invest (2013) 25:407-12. doi:10.1177/ 1040638713485228

8. Wilberts BL, Arruda PH, Kinyon JM, Frana TS, Wang C, Magstadt DR, et al. Investigation of the impact of increased dietary insoluble fiber through the feeding of distillers dried grains with solubles (DDGS) on the incidence and severity of Brachyspira-associated colitis in pigs. PLoS One (2014) 9:e114741. doi:10.1371/journal.pone.0114741

9. Caporaso JG, Lauber CL, Walters WA, Berg-Lyons D, Huntley J, Fierer N, et al. Ultra-high-throughput microbial community analysis on the Illumina HiSeq and MiSeq platforms. ISME J (2012) 6:1621-4. doi:10.1038/ismej. 2012.8

10. Caporaso JG, Kuczynski J, Stombaugh J, Bittinger K, Bushman FD, Costello EK, et al. QIIME allows analysis of high-throughput community sequencing data. Nat Method (2010) 7:335-6. doi:10.1038/nmeth.f.303

11. Segata N, Izard J, Waldron L, Gevers D, Miropolsky L, Garrett WS, et al. Metagenomic biomarker discovery and explanation. Genome Biol (2011) 12:R60. doi:10.1186/gb-2011-12-6-r60

\section{ETHICS STATEMENT}

All animal procedures were approved by the Institutional Animal Care and Use Committee of Iowa State University (Log Number: 1-12-7283).

\section{AUTHOR CONTRIBUTIONS}

Conceived and designed the experiments, analyzed the data, and contributed reagents/materials/analysis tools: EB and PP. Performed the experiments: PP and BA. Wrote the paper: EB.

\section{FUNDING}

This work was supported by a grant from the Iowa Pork Producers Association and the National Pork Board (NPB \#13-212). The funders had no involvement in study design, data collection, analysis and interpretation of data, writing the manuscript, or decision to submit the article for publication.

12. Lott SC, Voß B, Hess WR, Steglich C. CoVennTree: a new method for the comparative analysis of large datasets. Front Genet (2015) 6:43. doi:10.3389/ fgene.2015.00043

13. Benjamini $Y$, Hochberg Y. Controlling the false discovery rate: a practical and powerful approach to multiple testing. J R Stat Soc B (1995) 57:289-300.

14. Kelly J, Daly K, Moran AW, Ryan S, Bravo D, Shirazi-Beechey SP. Composition and diversity of mucosa-associated microbiota along the entire length of the pig gastrointestinal tract; dietary influences. Environ Microbiol (2017) 19:1425-38. doi:10.1111/1462-2920.13619

15. Chaban B, Links MG, Hill JE. A molecular enrichment strategy based on cpn60 for detection of epsilon-proteobacteria in the dog fecal microbiome. Microb Ecol (2012) 63:348-57. doi:10.1007/s00248-011-9931-7

16. Youmans BP, Ajami NJ, Jiang ZD, Campbell F, Wadsworth WD, Petrosino JF, et al. Characterization of the human gut microbiome during travelers' diarrhea. Gut Microbes (2015) 6:110-9. doi:10.1080/19490976.2015. 1019693

17. Costa MO, Chaban B, Harding JC, Hill JE. Characterization of the fecal microbiota of pigs before and after inoculation with "Brachyspira hampsonii". PLoS One (2014) 9:e106399. doi:10.1371/journal.pone.0106399

18. Koh HW, Kim MS, Lee JS, Kim H, Park SJ. Changes in the swine gut microbiota in response to porcine epidemic diarrhea infection. Microbes Environ (2015) 30:284-7. doi:10.1264/jsme2.ME15046

19. Yang Q, Huang X, Zhao S, Sun W, Yan Z, Wang P, et al. Structure and function of the fecal microbiota in diarrheic neonatal piglets. Front Microbiol (2017) 8:502. doi:10.3389/fmicb.2017.00502

20. Doyle LP. The etiology of swine dysentery. Am J Vet Res (1948) 9:50-1.

21. Burrough ER, Terhorst S, Sahin O, Zhang Q. Prevalence of Campylobacter spp. relative to other enteric pathogens in grow-finish pigs with diarrhea. Anaerobe (2013) 22:111-4. doi:10.1016/j.anaerobe.2013.06.004

22. Earley H, Lennon G, Balfe A, Kilcoyne M, Clyne M, Joshi L, et al. A preliminary study examining the binding capacity of Akkermansia muciniphila and Desulfovibrio spp., to colonic mucin in health and ulcerative colitis. PLoS One (2015) 10:e0135280. doi:10.1371/journal. pone. 0135280

23. Wilberts BL, Arruda PH, Kinyon JM, Madson DM, Frana TS, Burrough ER. Comparison of lesion severity, distribution, and colonic mucin expression in pigs with acute swine dysentery following oral inoculation with "Brachyspira hampsonii” or Brachyspira hyodysenteriae. Vet Pathol (2014) 51:1096-108. doi:10.1177/0300985813516646

24. Quintana-Hayashi MP, Mahu M, De Pauw N, Boyen F, Pasmans F, Martel A, et al. The levels of Brachyspira hyodysenteriae binding to porcine colonic mucins differ between individuals, and binding is increased to mucins 
from infected pigs with de novo MUC5AC synthesis. Infect Immun (2015) 83:1610-9. doi:10.1128/IAI.03073-14

25. Rowan F, Docherty NG, Murphy M, Murphy B, Calvin Coffey J, O'Connell PR. Desulfovibrio bacterial species are increased in ulcerative colitis. Dis Colon Rectum (2010) 53:1530-6. doi:10.1007/DCR. 0b013e3181fle620

26. Roediger WE, Moore J, Babidge W. Colonic sulfide in pathogenesis and treatment of ulcerative colitis. Dig Dis Sci (1997) 42:1571-9. doi:10.1023/ A:1018851723920

27. Hale VL, Chen J, Johnson S, Harrington SC, Yab TC, Smyrk TC, et al. Shifts in the fecal microbiota associated with adenomatous polyps. Cancer Epidemiol Biomarkers Prev (2017) 26:85-94. doi:10.1158/1055-9965. EPI-16-0337

28. Lau SK, Woo PC, Woo GK, Fung AM, Ngan AH, Song Y, et al. Bacteraemia caused by Anaerotruncus colihominis and emended description of the species. J Clin Pathol (2006) 59:748-52. doi:10.1136/jcp.2005.031773

29. Gophna U, Konikoff T, Nielsen HB. Oscillospira and related bacteria - from metagenomic species to metabolic features. Environ Microbiol (2017) 19:835-41. doi:10.1111/1462-2920.13658
30. Li XJ, Yue LY, Guan XF, Qiao SY. The adhesion of putative probiotic lactobacilli to cultured epithelial cells and porcine intestinal mucus. J Appl Microbiol (2008) 104:1082-91. doi:10.1111/j.1365-2672.2007.03636.x

31. Leonel AJ, Alvarez-Leite JI. Butyrate: implications for intestinal function. Curr Opin Clin Nutr Metab Care (2012) 15:474-9. doi:10.1097/MCO.0b013e32835665fa

32. Tamanai-Shacoori Z, Smida I, Bousarghin L, Loreal O, Meuric V, Fong SB, et al. Roseburia spp.: a marker of health? Future Microbiol (2017) 12:157-70. doi:10.2217/fmb-2016-0130

Conflict of Interest Statement: The authors declare that the research was conducted in the absence of any commercial or financial relationships that could be construed as a potential conflict of interest.

Copyright $\odot 2017$ Burrough, Arruda and Plummer. This is an open-access article distributed under the terms of the Creative Commons Attribution License (CC $B Y)$. The use, distribution or reproduction in other forums is permitted, provided the original author(s) or licensor are credited and that the original publication in this journal is cited, in accordance with accepted academic practice. No use, distribution or reproduction is permitted which does not comply with these terms. 